\title{
SEA: A PLATFORM FOR DESIGNING, CAPTURING AND PROCESSING LARGE-SCALE SURVEYS
} Alex Villazón, Vladimir Calderón and Ivan Krsul

\begin{abstract}
Collecting data through surveys is widely used by governments, international and non-governmental organizations to gather up-to-date and useful statistical information. Unfortunately, this process is tedious and often performed using paper support or customized software not allowing on-the-fly validations to avoid transcription errors at the early stage of the data collection. In this article, we describe SEA, a new web-based platform allowing sophisticated and large-scale surveys to be designed, captured and processed. SEA allows the survey designers to express complex input constraints through formulas for data validation. We describe the constraint language and the internals of the SEA engine, which is implemented using compilation, code generation and interpretation techniques.
\end{abstract}

Keywords: Code Generation, Compilation Techniques, Constraint Validation. 